\title{
Physics analysis of results of stochastic and classic stellarator coil optimization
}

\author{
Jim-Felix Lobsien* \\ E-mail: jim.lobsien@ipp.mpg.de \\ Michael Drevlak* \\ E-mail: michael.drevlak@ipp.mpg.de \\ Frank Jenko ${ }^{+}$ \\ E-mail: frank.jenko@ipp.mpg.de \\ Maurice Maurer ${ }^{+}$ \\ E-mail: maurice.maurer@ipp.mpg.de
}

Alejandro Bañon Navarro ${ }^{+}$

E-mail: alejandro.banon.navarro@ipp.mpg.de

Carolin Nührenberg*

E-mail: Carolin.Nuehrenberg@ipp.mpg.de

Thomas Sunn Pedersen*

E-mail: thomas.sunn.pedersen@ipp.mpg.de

Håkan M. Smith*

E-mail: hakan.smith@ipp.mpg.de

Yuriy Turkin*

E-mail: yuriy.turkin@ipp.mpg.de

\section{W7-X Team}

*Max-Planck Institute for Plasma Physics, Greifswald, Germany

${ }^{+}$Max-Planck Institute for Plasma Physics, Garching, Germany

Abstract. In this paper we follow up on the results from our previous publication [Lobsien J, et al., Nucl. Fus., Vol 58 (2018)106013], where it was found that stellarator coil design optimization can be substantially improved by using a stochastic optimisation approach. In that paper performance was quantified by lower (better) and more narrow (more robust) distributions of the penalty functions at the end of 
the optimisations. Here, we evaluate and compare the various coil sets of the previous paper but seek a verification and deeper understanding of the physics performance by replacing the relatively simple penalty function estimate with more accurate ones from state-of-the-art calculations of MHD stability, neoclassical transport in the $1 / \nu$-regime, fast particle confinement, and gyrokinetic behavior. The investigation shows that stochastic stellarator coil optimization generally outperforms the earlier non-stochastic stellarator optimization, also when using these more accurate metrics, generally confirming and quantifying the better performance. We do find some discrepancies, indicating that the penalty function does not represent a physics performance optimum perfectly. For example, as pointed out by others before us, the depth of the magnetic well is not a sufficiently good proxy for MHD stability, and the neoclassical transport can be significantly reduced in configurations that have relatively high field errors and therefore high penalty values. Thus, our work points to areas where better physics model inside the optimisation loop are needed, than what is currently represented by our penalty function.

Keywords: Stellarator, Coil Optimization, Engineering Tolerances, Robust Magnetic Field, Stochastic Optimization, MHD, Neoclassical Transport, Fast-Particle Confinement, Turbulent Transport

Submitted to: Nucl. Fusion

\section{Introduction}

A stellarator is defined by a set of nested toroidal flux surfaces which may be optimized to fulfill multiple performance criteria. In the case of Wendelstein 7-X (W7-X) these criteria were summarized in the W7-X objectives [3], which not only concentrated on the quality of the vacuum magnetic surfaces but also focused on the properties of finite-pressure equilibria. Special emphasis was placed on a small Shafranov shift, good MHD stability properties, a small neoclassical transport, a general reduction of parallel currents, and good $\alpha$-particle confinement in fusion-relevant operating regimes. All these properties are determined by the shape of the plasma boundary, because this outermost flux surface defines the magnetic field in its interior, which in turn defines the corresponding behaviour when a plasma pressure is present. The design of a stellarator, therefore, may begin with the shape of the plasma boundary and continues with the optimization of a set of finite current-carrying filaments outside the plasma, which is meant to produce the desired magnetic field. The starting point is usually a first guess of the coil structure computed with NESCOIL [4] or REGCOIL [5]. Each coil is then parametrised by either Fourier coefficients or spline points and a penalty function $f$ is defined which measures the difference between the magnetic field produced by the coils and the desired vacuum magnetic field of the stellarator called the target magnetic field. Nonlinear coil optimization, as done with codes like ONSET [1], COILOPT ${ }^{++}$[7] and FOCUS [8], then minimizes this nonlinear function $f$, which takes the coil parameters 
as an input value and summarizes the fitness of the corresponding magnetic field in a real value $y \in \mathbb{R}$. The fitness is characterized by a set of quality criteria of which the field error is the most dominant one. It is quantified by $\mathbf{B} \cdot \mathbf{n}$, where $\mathbf{B}$ is the vector of the magnetic field produced by the coils and $\mathbf{n}$ is the normal on the plasma boundary. In general, $\mathbf{B}$ may include internal plasma currents, which were absent in the optimization of W7-X. The minimization of the field error is one major challenge of stellarator coil optimization but, from a theoretical point of view, deviations from the ideal target are unavoidable due to the discrete nature of the coil set which inevitably produces a corresponding ripple of the magnetic surfaces. Additionally, deviations occur due to geometric constraints necessary for the manufacturing of the coils and assembly of the coil set.

Once the coil optimization arrives at the lower boundary of the field error, the penalty function $f$ is extended by additional quality criteria and the optimization continues. Each new set of quality criteria describes a more intricate property of the set of nested toroidal flux surfaces which define the stellarator. But the more intricate the quality criterion, e.g. effects with finite plasma pressure, the more time it takes to compute a single evaluation of $f$. Therefore, it is the challenge of nonlinear coil optimization to include new elements within the penalty function $f$ step by step, such that the field error is not increasing while keeping the complexity of the quality criteria in $f$ moderate so as to avoid excessive computing times.

Traditionally, the coil optimization is completed when the underlying magnetic field fulfills the same performance criteria, that initially led to the shape of the plasma boundary. But optimizing them requires a transition of the optimization targets. The optimization of the quality criteria, which reduces the difference between the magnetic field from the coils and the magnetic field defined by the plasma boundary, is replaced by the direct optimization of the performance of the magnetic field produced by the coils. This last step is similar to the optimization of the target stellarator magnetic field itself, with the difference that instead of varying the plasma boundary one changes the coil configuration that defines the plasma boundary.

Once a suitable set of filaments has been found, the focus is changed and the coil configuration is evaluated in a perturbation analysis. Each deviation leads to a change of the magnetic field and consequently to a change in the performance criteria. After performing a statistical ensemble of deviations the perturbation analysis defines a measure of the change of the quality of the magnetic field w.r.t. deformations of the coil set. A prescribed lowest acceptable magnetic field quality then leads to coil construction tolerances which will require more time and resources the lower they are. Unfortunately, recent major stellarator projects have had the tendency to be negatively influenced by their own strict tolerance requirements, e.g. Wendelstein 7-X [9] and NCSX [10].

With the aim to ease the construction of future stellarator projects, we established a stochastic version of stellarator coil optimization that was able to increase the construction tolerances during the design process of the coil configuration [2]. Nonlinear coil optimization is combined with an iterative perturbation analysis with the result 
that the target magnetic field is more accurately reproduced and is also more resilient against coil displacements w.r.t. the penalty function $f$. The new technique replaces the optimization of a single coil configuration $f_{1}$ with the optimization of a cloud of neighboring coil configurations $f_{N}$. Each element of the cloud is a perturbation of the original coil configuration at the cloud's center. The cloud is characterized by the number of samples $N$ (number of perturbations) and their Gaussian distribution around the unperturbed configuration. With the aim to test the stochastic version of stellarator coil optimization against its classical counterpart, a compact optimization sequence for the original W7-X plasma boundary [11] was developed, in which the complexity of the penalty function stayed rather low. The test included 6 different sample sizes (1, 100, 1000, 2000, 4000 and 8000) and compared the results based on their fitness and robustness w.r.t. $f$. Here, $f_{1}$ is the classic, single coil optimization case. The algorithm together with the optimization sequence is described in more detail in [2]. The 6 optimization runs of the optimization sequence concentrate mainly on the field error and at the end on properties of the vacuum magnetic field, while adhering the geometric constraints throughout the optimization. The penalty values at the end of the optimization sequence resulted in the following ordering of the sample size cases

$$
f_{8000}<f_{4000}<f_{2000}<f_{1}<f_{1000} \ll f_{100},
$$

where the biggest gap in this order is between the case with 1000 and 100 samples. The case with 8000 samples has a $20 \%$ lower penalty value compared to the coil optimization that uses just a single sample. In all cases, the penalty on the field error constitutes $85 \%$ of the total penalty value since the design values of the remaining quality criteria are reached with quite high accuracy. The focus of the first study was to demonstrate the advantages of stochastic optimization in the context of stellarator coil design, using only ONSET. The improved results do not necessarily imply that these results are superior to newer coil design tools (such as FOCUS), only that the stochastic optimization itself was beneficial.

During the optimization and subsequent perturbation analysis in [2], the fitness of the coil sets and the corresponding quality of the magnetic fields were measured by the penalty function $f$. This perspective is limited and shows only the differences w.r.t. the target magnetic field and not how these magnetic field differences influence the more fundamental physics properties of the configuration. In this paper we move beyond the scope of $f$ and compare the coil sets optimized in [2] by the actual performance criteria which lead to the shape of the plasma boundary. We shift the perspective, from investigating properties of magnetic fields to a physics analysis of finite- $\langle\beta\rangle$ equilibria. This way, we make a better assessment of the actual fitness of the coil sets and test the ordering obtained from the penalty values in equation (1) against the number of samples used during the optimization. Loosely formulated, we try to assess how much the lower penalty function has brought us in terms of more fundamental and important physics properties, assessed with the best tools we have available today. It is at least in principle possible that the significantly reduced penalty value in the end does not 
translate into significantly improved physics properties. So part of our purpose is also to make a first assessment of the appropriateness of the penalty function itself, although admittedly the data presented here are too sparse to give more than a first indication.

In this physics analysis, the single coil optimization with a single sample is the reference case of classic coil optimization and we refer to the rest of the cases optimized in [2] as stochastic case $N$. All the results are ranked with the target magnetic field, defined by the original W7-X boundary [12]. The origin of all magnetic fields is explained in more detail in section 2 and their differences w.r.t. the quality criteria used at the end of the optimization are described in section 3. The performance criteria are chosen following the list of the W7-X objectives in [3]. We describe the development of the Shafranov shift in section 4.1 and the global ideal MHD stability properties using the CAS3D stability code [15] in section 4.2. The neoclassical transport will be discussed in section 4.3, and the confinement of fast particles using the ANTS code [16] will be compared in section 4.4. Last but not least, we move beyond the performance criteria for which the W7-X boundary was optimized and present a mode analysis in section 4.5 showing a glimpse into the gyrokinetic behaviour observable in a selection of three cases.

\section{Origin of the Magnetic Fields}

The optimization that led to the design of the actual W7-X coil set can unfortunately not be reproduced today. That optimization procedure involved iterations and constraints that are not easily automated and not sufficiently documented. Some of these are described below. The optimization, which compared traditional and stochastic stellarator coil optimization used the original W7-X "high-mirror" configuration as the target magnetic field. The boundary was first introduced in [12] but is better described in [20]. The coil configuration that reproduced the magnetic fields of the original W7-X configurational space is no longer available and its shape has repeatedly changed since its first publication in [17]. The main motivation was the manufacturability at the time combined with the additional desire of more experimental flexibility. With the change of the coil configuration the magnetic fields of the W7-X configurational space changed. Consequently, there is a not fully known difference between the original target magnetic field and the magnetic field for which the coils in the end were designed.

We computed an analytic current distribution on a surface outside the plasma boundary with NESCOIL [4], that reproduces the original boundary with acceptable precision. We used the magnetic field produced by this current sheet to obtain the target values used for the optimization and therefore, we refer to this magnetic field as the target magnetic field. Choosing a vanishing net toroidal coil current guarantees that the magnetic field of the stellarator can be generated from poloidally closed magnets, and that there is no need for toroidal or helical magnets. This way, a discretization of the current sheet into poloidally closed modular current filaments is possible. These provide the starting point of the coil optimization with ONSET [1] and its stochastic 
extension [2]. The optimization ended after 6 runs with the coil sets that produce the magnetic fields we refer to as the reference case and the stochastic cases.

The finite- $\langle\beta\rangle$ MHD equilibria of the 7 magnetic fields are computed with the free boundary version of VMEC [18] [19], where we use the pressure profile of the stability analysis [20] of the original W7-X configurational space [11] which is proportional to

$$
p(\rho) \propto 7-11 \rho^{2}+4 \rho^{4} \quad \text {, where } \quad 0 \leq \rho \leq 1
$$

is the minor radius normalized to the minor radius of the last closed flux surface. VMEC is used with 65 flux surfaces allowing residual forces of the order of $10^{-11}$. The resolution of the MGRID files uses 130 points in radial- and z-direction and 62 points in $\varphi$-direction. Here we denote that the optimization of the finite- $\langle\beta\rangle$ effects of the original W7-X boundary was done using the fixed-boundary VMEC. This means that the free-boundary VMEC equilibrium of the target magnetic field was never directly optimized, only indirectly through the fixed-boundary VMEC studies.

\section{Quality Criteria}

The quality criteria used during the optimization are visualized in figure 1 and the corresponding values of the stochastic and reference cases together with the target values are listed in table A1 of the Appendix A. We added the corresponding penalty values to better assess equation (1). During the optimization, the squared residual of each quality criterion w.r.t. its corresponding target value was a weighted part in the penalty function $f$ whose minimization was the main task of the optimization sequence described in [2].

The field error is quantified with two values, the maximum local field error $\max e_{l}=\frac{|\mathbf{B} \cdot \mathbf{n}|}{|\mathbf{B}|}$, where $\mathbf{B}$ is the vector of the magnetic field and $\mathbf{n}$ is the normal on the plasma boundary, and the average global field error $e_{a}=\frac{\int_{A} e_{l} d A}{A}$, where $A$ is the area of the plasma boundary. They are the most dominant quality criteria in the penalty function directly quantifying the difference between the target magnetic field and the magnetic field produced by the coils. The target magnetic field is produced by an analytic current distribution computed with NESCOIL (12 poloidal and 10 toroidal modes) on a current-carrying surface $30 \mathrm{~cm}$ outside the plasma boundary. Its field error sets the lower boundary compared to the field errors of the coil optimization study as can be seen in table 1 . 


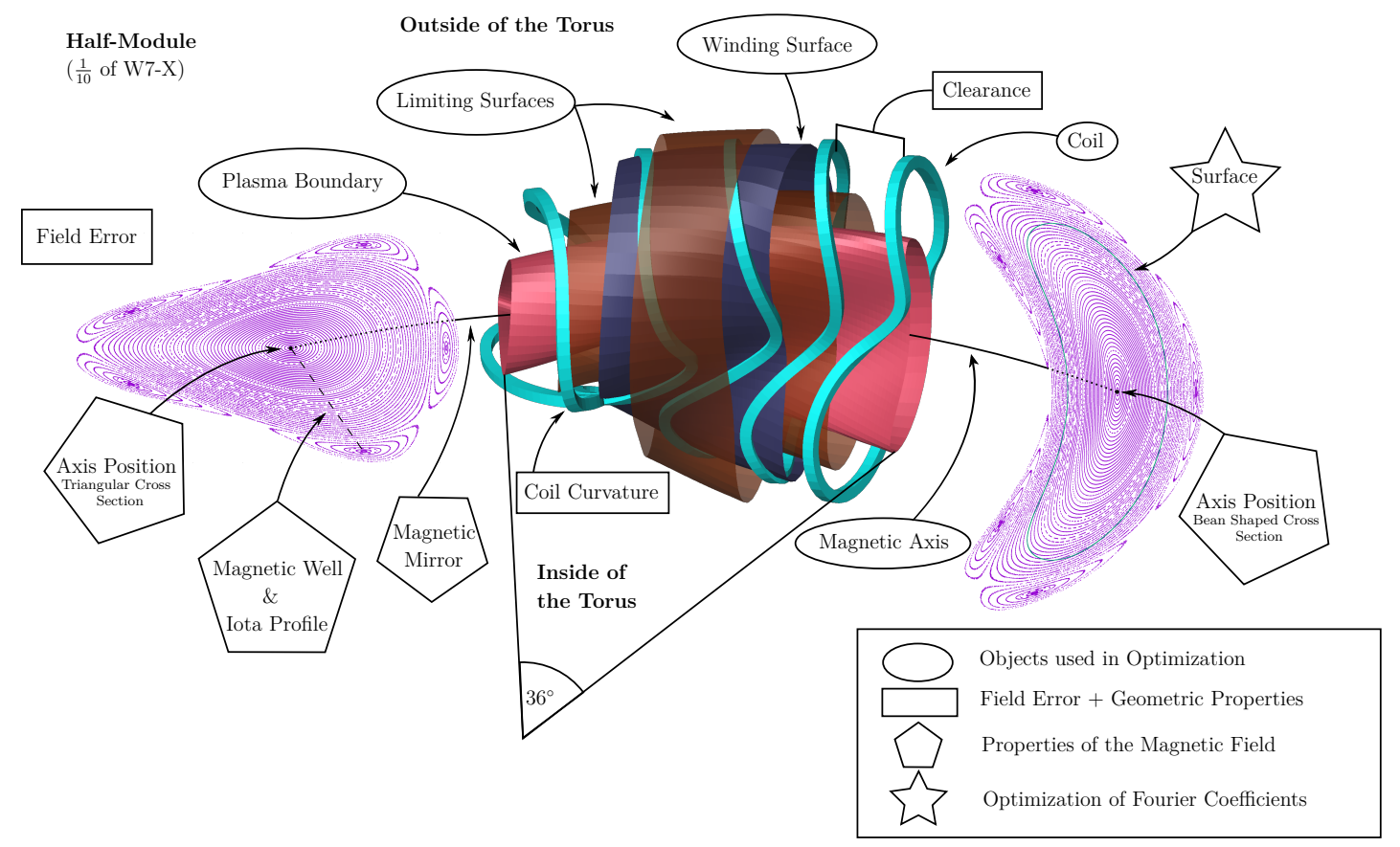

Figure 1: A W7-X half-module is shown together with the surfaces used during a stellarator coil optimization with ONSET [1]. Additionally, we present the quality criteria used during the compact optimization sequence cf.A1.

Table 1: Values of the Field Error

\begin{tabular}{lcc}
\hline Magnetic Fields: & $\begin{array}{c}\text { Maximum Local } \\
\text { Field Error: }\end{array}$ & $\begin{array}{c}\text { Average Global } \\
\text { Field Error: }\end{array}$ \\
\hline Target Magnetic Field & $3.4 \times 10^{-4}$ & $9.5 \times 10^{-5}$ \\
Stochastic Case 8000 & $6.0 \times 10^{-2}$ & $1.6 \times 10^{-2}$ \\
Stochastic Case 4000 & $5.7 \times 10^{-2}$ & $1.66 \times 10^{-2}$ \\
Stochastic Case 2000 & $6.0 \times 10^{-2}$ & $1.95 \times 10^{-2}$ \\
Reference Case & $7.0 \times 10^{-2}$ & $1.7 \times 10^{-2}$ \\
Stochastic Case 1000 & $7.0 \times 10^{-2}$ & $1.92 \times 10^{-2}$ \\
Stochastic Case 100 & $8.0 \times 10^{-2}$ & $2.27 \times 10^{-2}$ \\
\hline
\end{tabular}

The differences in the field error between the optimized coil cases reflect the order of the penalty values in equation (1) obtained after the optimization and highlights that the stochastic case 8000 has nearly the same field error as the stochastic case 4000, that the reference case is somewhere between the stochastic case 2000 and stochastic case 1000 and that the worst values with the largest gap among the coil cases has the stochastic case 100. The vacuum Poincare plots of all the magnetic fields at the bean-shaped cross section presented in figure 2 confirm this situation and show that the magnetic field of the stochastic case 100 deviates most from the target magnetic field. But variations in the Poincare plots do not necessarily lead to changes in the plasma performance as stated in [13] and [14]. Table A1 visualizes in numbers that all coil configurations show 
quite good agreement on the properties of the vacuum magnetic field and meet the geometric constraints such that all coil configurations are valid approximations of the target magnetic field.

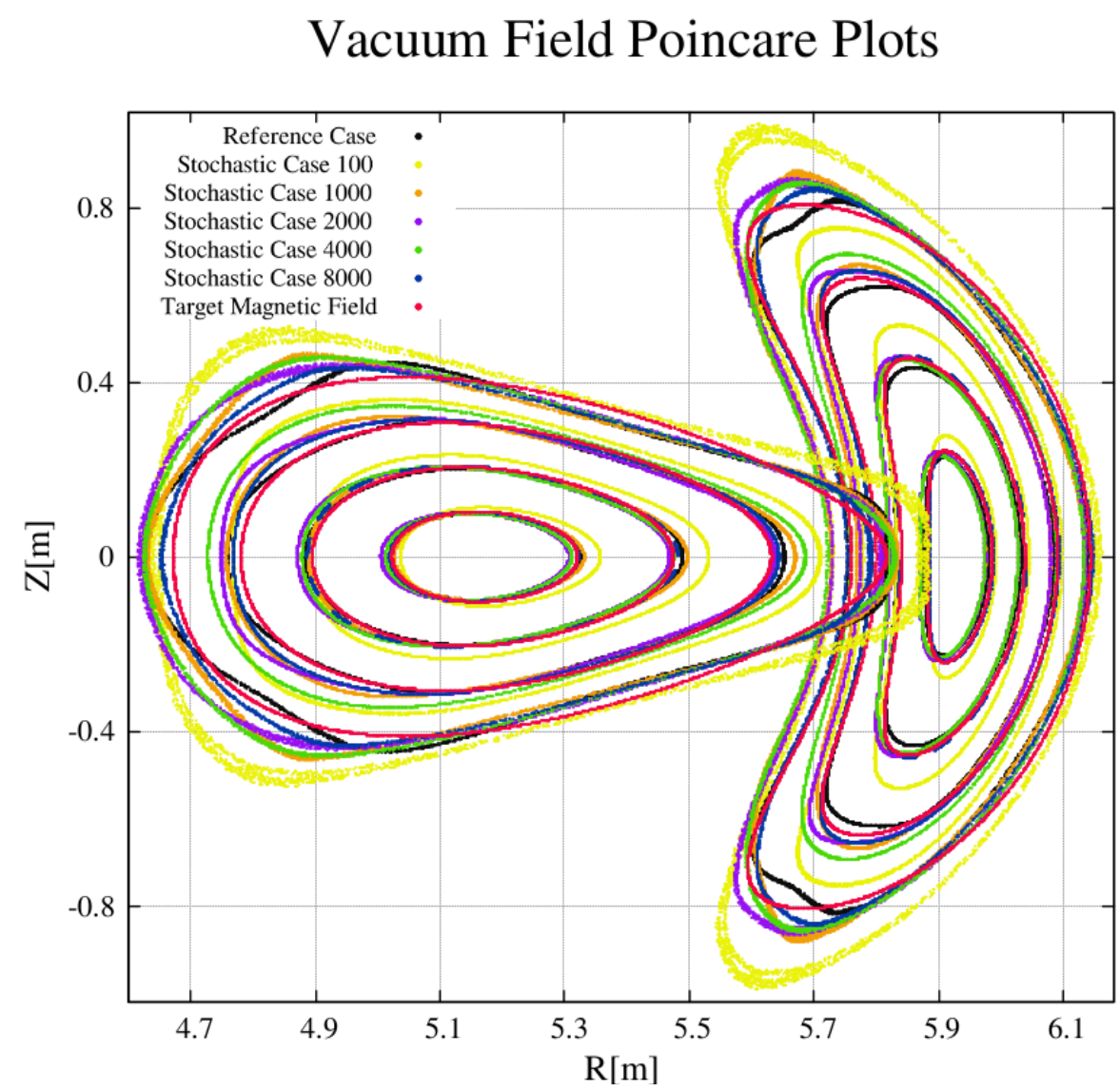

Figure 2: Poincare plots of the vacuum field at the bean-shaped and triangular cross section

The geometric properties of the filament structure guarantee the feasibility of the stellarator construction. The material used and consequently the extent of the coil usually defines the maximal allowed coil curvature. It is defined as $\kappa=\frac{1}{R}$, where $R$ is the radius of the smallest circle representing a coil segment. Additionally, one tries to avoid unnecessary undulations, which is enforced by the second curvature. It is a weighted integral of the first curvature and fully described in [22]. The finite extent of the coil also defines the required clearance between adjacent coils, and the two limiting surfaces guarantee that the coils do not get too close or too far away from the plasma boundary. Besides these properties, coil to plasma separation and coil length ([5], [6], [8], [21]) are often used as design metrics.

The basic properties of the magnetic field are characterized by 7 aspects in the penalty function:

- The magnetic axis is determined at the beginning and at the end of the half-module.

- The difference between the magnetic field strength on the axis at the start and at the 
end of the half-module is referred to as the magnetic mirror. It is normalized to the sum of the two magnetic field strength values. It is important for the confinement of fast particles and will be discussed in section 4.4 .

- The value of iota on the axis and the shear is determined. The latter is computed by taking the difference of iota on axis and iota $0.2 \mathrm{~m}$ off axis at the beginning of the half-module at $z=0$.

- The change of the magnetic volume along the radial direction is summarized in the magnetic well, which is necessary for the global MHD stability and will be discussed in section 4.2 .

- The Fourier coefficients of inner flux surfaces can be computed and optimized towards the coefficients of the corresponding surfaces of the target magnetic field. They are derived in PEST coordinates [45] and we chose the $R_{m n}$ and $Z_{m n}$ of a flux surface slightly inside the plasma boundary.

\subsection{Remark:}

We point out, that only the values of first curvature, the clearance and the two axes positions have physical units.

\section{Performance Criteria}

\subsection{Shafranov Shift}

The Shafranov shift measures the deviation of the magnetic axis when a plasma pressure is applied. The reduction of the Shafranov shift was one of the key objectives in the design of W7-X [3].

In figure 3 we compare the position of the magnetic axis at both up-down symmetric plasma cross sections as a function of normalized plasma pressure $(\langle\beta\rangle)$. The Shafranov shift between $\langle\beta\rangle=0 \%$ and $\langle\beta\rangle=5 \%$ measured at the bean-shaped cross section is about $1 \mathrm{~cm}$ lower for the stochastic case 100 compared to the rest of the cases. They all have almost the same shift with the reference case having the largest one. At the triangular cross section the stochastic case 100 has again the smallest Shafranov shift, which is this time $2 \mathrm{~cm}$ smaller than the Shafranov shift of the reference case. The rest of the cases including the target magnetic field have a Shafranov shift in between the latter two cases, around $1 \mathrm{~cm}$ higher than the stochastic case 100 and around $1 \mathrm{~cm}$ lower than the reference case. The Shafranov shift is in general a factor of two lower at the bean-shaped cross section compared to the triangular cross section due to the different elongations of the two cross sections. The results yield the following ordering:

$$
f_{100}<\text { Target } \approx f_{8000} \approx f_{4000} \approx f_{2000} \approx f_{1000}<f_{1} .
$$


Bean Shaped Cross Section

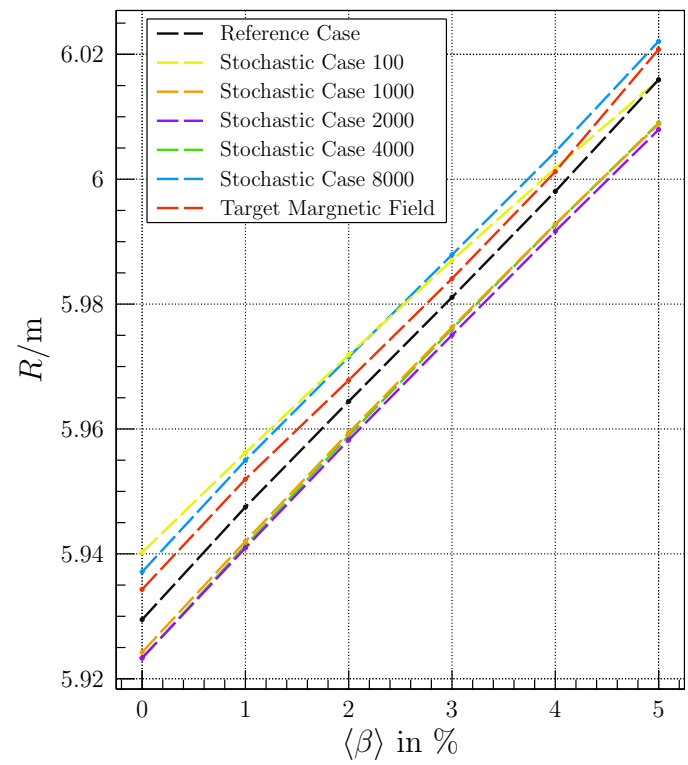

Triangular Cross Section

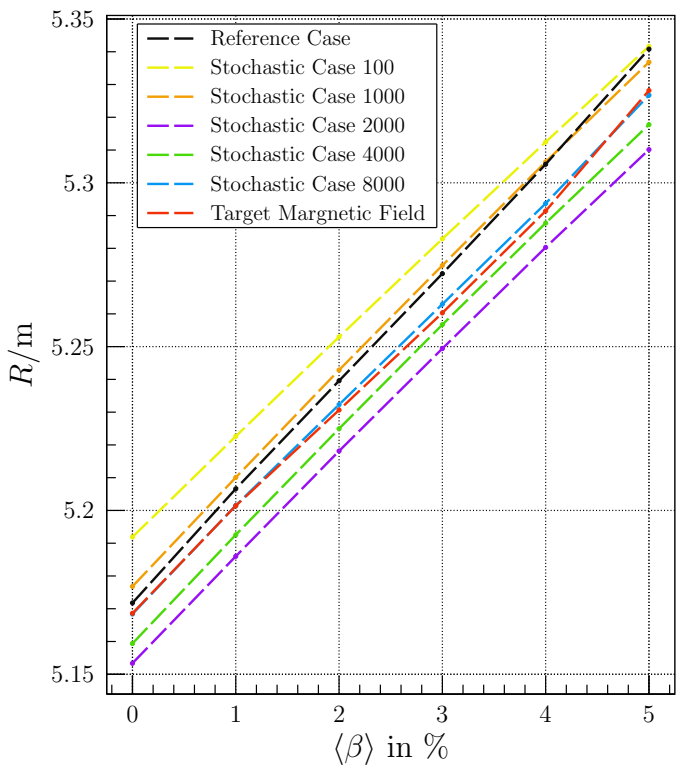

Figure 3: Shift of the magnetic axis from $\langle\beta\rangle=0 \%$ up to $\langle\beta\rangle=5 \%$ measured at both ends of the half-module.

\subsection{Stability}

The depth of the magnetic well is a figure of merit in the ideal MHD considerations [23]. Having a magnetic well means that the rate of increase of the contained magnetic volume with toroidal flux,$V^{\prime}(s)$, decreases with radius, i.e. $V^{\prime \prime}(s)<0$ [24]. In ONSET, the magnetic well is defined as the normalized difference of the specific volume of two well-separated magnetic surfaces (including the magnetic axis)

$$
-2 \cdot \frac{V_{2}^{\prime}-V_{1}^{\prime}}{V_{1}^{\prime}+V_{2}^{\prime}}
$$

Here $V_{1}^{\prime}$ is the specific volume belonging to the magnetic surface closer to the magnetic axis and $V_{2}^{\prime}$ the corresponding specific volume belonging to the magnetic surface closer to the last closed flux surface. During our optimization we computed $V_{1}^{\prime}$ on the magnetic axis and $V_{2}^{\prime}$ on the surface at $\rho=0.2$, where $\rho$ is the normalized minor radius. The specific magnetic volume is computed as the average line integral along a magnetic field line normalized by the magnetic field strength and is expressed as the limit of

$$
\lim _{N \rightarrow \infty} \frac{1}{N} \int_{N} \frac{d l}{B}=\frac{d V}{d \Psi} \quad \text { with } \quad \Psi-\text { toroidal flux. }
$$

The target magnetic field provides the design value of the magnetic well of $7.0 \times 10^{-3}$. The stochastic cases $4000 \& 8000$ possess a deeper magnetic well of $\approx 8 \times 10^{-3}$ and the reference case has a more shallow magnetic well of $5.4 \times 10^{-3}$. The magnetic well of the stochastic cases $100 \& 1000 \& 2000$ is more than two times deeper than the magnetic well of the stochastic cases $4000 \& 8000$. Having a magnetic well is a necessary condition 
for global ideal MHD stability and the result suggests that the stochastic cases are more stable than the reference coil case.

Plasma Cross-sections

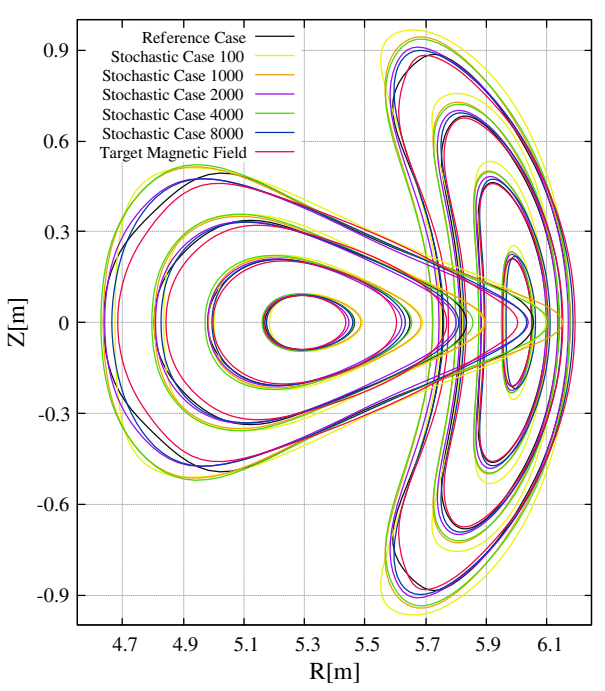

Ideal MHD Eigenvalues

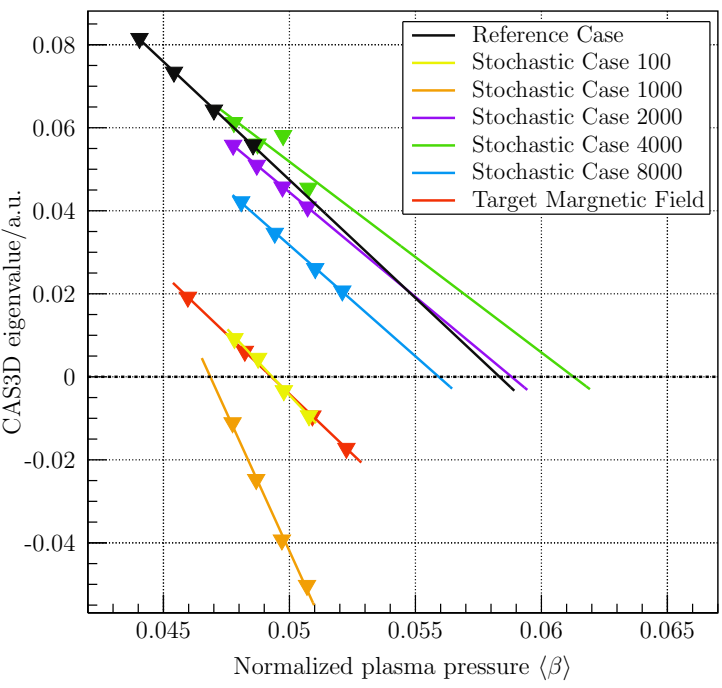

Figure 4: Left: Cross sections of the equilibria at $\langle\beta\rangle=0.048$ at the triangular- and beanshaped cross section. Right: Ideal MHD eigenvalue calculations versus average plasma- $\beta$.

We discuss the global ballooning stability of the reference and stochastic cases and compare the results with the target magnetic field based on four MHD equilibria each. The volume-averaged plasma- $\beta$ is varied in a small range around the W7-X beta stability limit of about $5 \%$. The rotational transform varies between $\iota=5 / 6$ near the magnetic axis and $\iota=5 / 5$ near the plasma boundary in the reference and the stochastic cases. Only in the target magnetic field we see $\iota<0.8$ on the axis. For the equilibria at $\langle\beta\rangle=0.048$ two characteristic plasma cross sections of one half-module are shown in figure 4 (left).

The ideal MHD computations were done using the CAS3D code [15] with 520 Fourier harmonics for the scalar perturbation components and 64 equidistant flux intervals for the radial discretization. We consider even-parity fixed-boundary perturbations for which the normal displacement is up-down symmetric on the up-down symmetric plasma cross sections, i.e. at the bean-shaped and triangular cross sections, figure 4 (left). The angular resolution is reduced by using a phase-factor transform that extracts a potentially strongly varying part of the scalar perturbation components.

Global ballooning stability prevails for the $\beta$-values studied of the reference case and stochastic cases $8000 \& 4000 \& 2000$ as can be seen from their positive eigenvalues in figure 4 (right). The target magnetic field together with the stochastic cases $100 \& 1000$ have unstable eigenvalues for the poloidal mode number $\approx 100$ considered in figure 4 . Here, extrapolation from the stable or unstable side yields points of marginal stability. Above the marginal plasma- $\beta$ the equilibrium is predicted to be unstable. Hence looking 
at the modes with poloidal mode number $\approx 100$, we can derive the stability limit of all the cases shown in table 2 .

Table 2: Stability limit of poloidal mode number 100

\begin{tabular}{lc}
\hline Magnetic Fields: & Stability Limit: \\
\hline Stochastic Case 1000 & $\langle\beta\rangle=4.7 \%$ \\
Stochastic Case 100 & $\langle\beta\rangle=4.9 \%$ \\
Target Magnetic Field & $\langle\beta\rangle=4.9 \%$ \\
Stochastic Case 8000 & $\langle\beta\rangle=5.6 \%$ \\
Reference Case & $\langle\beta\rangle=5.8 \%$ \\
Stochastic Case 2000 & $\langle\beta\rangle=5.9 \%$ \\
Stochastic Case 4000 & $\langle\beta\rangle=6.1 \%$ \\
\hline
\end{tabular}

Higher mode numbers give more stringent stability limits, but the respective spatial structure is of very small scale. The wavelength of the poloidal mode number $\approx 100$ is only one order of magnitude above the Larmor-radius of gyrating ions moving in the corresponding magnetic field. Therefore, the spatial structure is close to the point where physics beyond ideal MHD should be taken into account.

The MHD ballooning stability properties of the reference case and the stochastic cases $2000 \& 4000 \& 8000$ are almost the same and somehow more stable than the target magnetic field. Even though the latter cases are mere approximations of its vacuum magnetic field their rotational transform profile has less shear than that of the the target magnetic field at finite plasma- $\langle\beta\rangle$. The stochastic case 100 is as stable as the target magnetic field and only the stochastic case 1000 is less stable. The maximum amplitudes of the normal-displacement harmonics are located quite close to the edge in the stochastic cases $100 \& 1000$ for which, near the plasma boundary, the local stability Mercier criterion is violated, too. Therefore, we additionally looked at medium-modenumber free-boundary perturbations and compared the stability limits of the poloidal mode number $\approx 30$. This new perspective does not change the order obtained before and just decreases the values of the stochastic cases $100 \& 1000$ while increasing the values of the rest.

The values of the magnetic well are insufficient indicators, since they indicate a greater difference between the stochastic and reference cases. Here we point out that the optimization leading to the W7-X configurational space was based on fixed-boundary equilibria and that MHD stability properties entered the target function by evaluation of local stability criteria (Mercier and resistive interchange) or driving terms (local fieldline ballooning) [11]. Nevertheless, both investigations arrive at a similar $\langle\beta\rangle$-limit. Besides the vacuum-field magnetic well, other equilibrium properties are important in global ideal MHD stability, e.g. the rotational transform profile. Falling below iota $=5 / 6$ in the target magnetic field in part explains why a decrease in vacuum-field magnetic 
well does not result in an increased $\langle\beta\rangle$-stability limit. The final order is:

$$
f_{4000}<f_{2000}<f_{1}<f_{8000}<\text { Target }<f_{100}<f_{1000} .
$$

\subsection{Neoclassical Transport}

The neoclassical confinement at low collisionalities in the $1 / \nu$-regime can be characterized by the 'effective helical ripple' $\epsilon_{\text {eff }}$ (see [25] and references therein). This quantity can be determined efficiently from an analytic solution of the bounce-averaged drift kinetic equation to calculate the neoclassical transport coefficients without using a simplified model of the magnetic field [26].

$$
\langle\beta\rangle=5 \%
$$

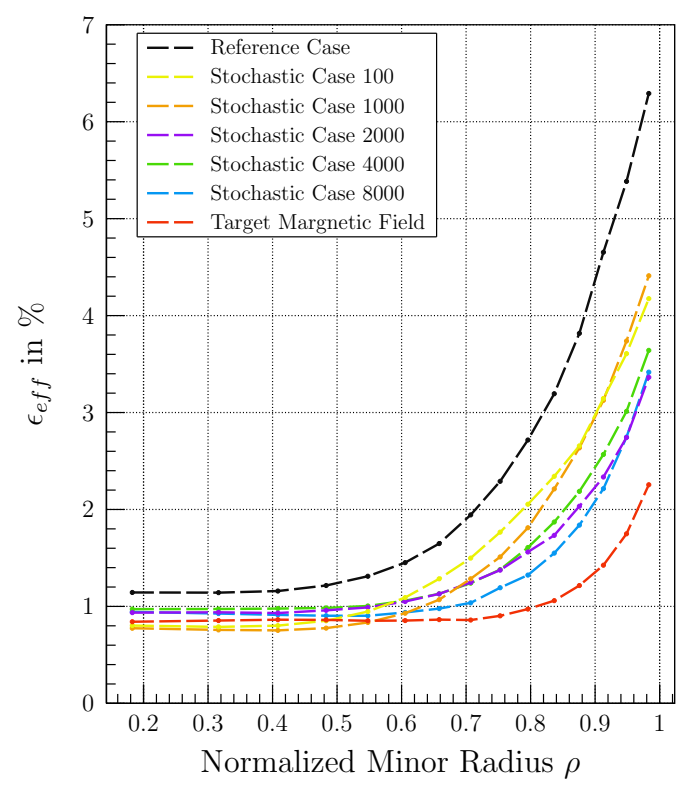

Figure 5: Profile of epsilon effective at $\langle\beta\rangle=5 \%$

In figure 5 we illustrate the $\epsilon_{\text {eff }}$ profile of all 7 cases at $\langle\beta\rangle=5 \%$. The radial profile is in all cases quite similar and only differs in the initial value and the ascent towards the edge. The target magnetic field reaches on average over the whole radial extent the lowest $\epsilon_{\text {eff }}$ profile. Its performance is only rivalled by the stochastic cases $100 \& 1000$ close to the magnetic axis. Their profile, in turn, has a steep ascent towards the edge which is comparable to the ascent of the reference case which has by far the highest $\epsilon_{\text {eff }}$ values. The $2^{\text {nd }}$ best $\epsilon_{\text {eff }}$ performance has the stochastic case 8000 which is closely followed by the stochastic cases $2000 \& 4000$.

The magnetic field in Boozer coordinates can be described by Fourier coefficients $b_{m n}$. The Shafranov shift reduces the magnetic mirror term $b_{01}$ when increasing $\langle\beta\rangle$ from $0 \%$ to $5 \%$ which leads in all cases to a decrease of $\epsilon_{\text {eff }}$ close to the axis [27]. The second consequence of the Shafranov shift is a general increase of the magnitude of the higher Fourier harmonics which leads to an increased transport gradient at the edge. 


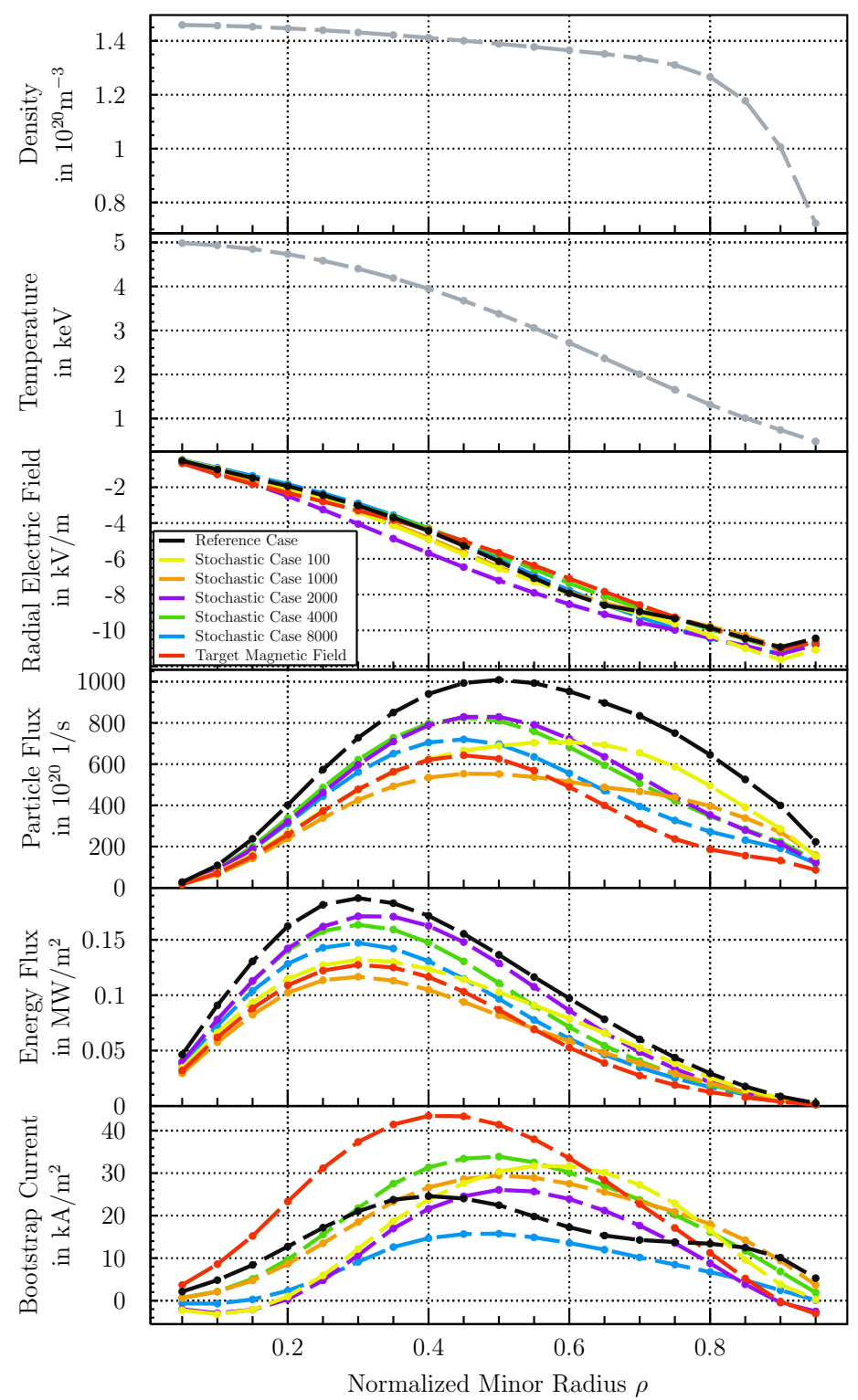

Figure 6: The neoclassical transport is shown for the density and temperature distribution displayed in the top first and top second picture. From third top to bottom the radial electric field, particle and energy flux and the bootstrap current is displayed all along the radial direction.

In figure 6 we present neoclassical transport calculations based on the 'Drift Kinetic Equation Solver' DKES [28] [29]. In order to reach $\langle\beta\rangle=5 \%$ we chose a density of $1.46 \times 10^{20} \mathrm{~m}^{-3}$ and a temperature of $5 \mathrm{keV}$ in the core. The corresponding profiles are shown in the first and second frame of figure 6 . Their shape is chosen similar to profiles found during the experimental campaign OP1.2 of W7-X while the overall pressure profile reaches $\langle\beta\rangle=5 \%$. A multiplication of the density and temperature profile yields the pressure profile defined in (2), which was used for the equilibria calculations with the free boundary version of VMEC [19]. The radial electric field, the energy and particle 
fluxes together with the bootstrap current along the radial direction are shown in figure 6 (third to bottom frame, respectively). There is almost no difference in the radial electric field, but the development of the particle and energy flux in the radial direction

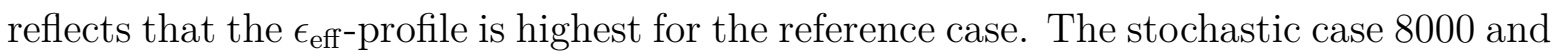
the target magnetic field have quite similar energy flux development but are noticeably lower than the reference case. The stochastic cases $100 \& 1000$ have the same particle and energy flux as the target magnetic field close to the core but diverge towards the edge. The particle and energy flux development of the stochastic cases $2000 \& 4000$ lies in between the reference case and the stochastic case 8000. A different picture presents itself regarding the development of the bootstrap current as can be seen in table 3, where we integrated the bootstrap current density over the cross sectional area.

Table 3: Integrated bootstrap current density over the cross sectional area.

\begin{tabular}{lc}
\hline Magnetic Fields: & Bootstrap Current \\
\hline Stochastic Case 8000 & $17 \mathrm{kA}$ \\
Stochastic Case 2000 & $22 \mathrm{kA}$ \\
Reference Case & $28 \mathrm{kA}$ \\
Stochastic Case 100 & $31 \mathrm{kA}$ \\
Stochastic Case 1000 & $32 \mathrm{kA}$ \\
Stochastic Case 4000 & $33 \mathrm{kA}$ \\
Target Magnetic Field & $35 \mathrm{kA}$ \\
\hline
\end{tabular}

The stochastic case 8000 has the lowest total bootstrap current, the reference case is situated in the middle and the highest bootstrap current is found surprisingly in the target magnetic field. A calculation of the equivalent bootstrap current of an elongated tokamak (corr. scale found in [31]) with the same volume and aspect ratio with NTSS [32] yields values around $220 \mathrm{kA}$. The bootstrap current of a more realistic circular scaled tokamak with the same volume and aspect ratio would yield values around $600 \mathrm{kA}$. Consequently, the bootstrap current is significantly reduced in all 7 cases considered, which confirms the minimization of the bootstrap current of the HELIAS line described in [30]. The small differences in total bootstrap current between the cases can thus be neglected.

In summary, the neoclassical confinement is best in the target magnetic field and closely followed by the stochastic case $8000 \& 1000$, which have almost the same performance. The rest of the stochastic cases have worse neoclassical confinement although still better than the performance of the reference case, yielding the following order:

$$
\text { Target }<f_{8000} \approx f_{100}<f_{4000} \approx f_{1000}<f_{2000}<f_{1}
$$




\subsection{Fast Particle Confinement}

In the case of W7-X a magnetic mirror of $10 \%$ or higher is a necessary condition to assure good $\alpha$-particle confinement [33]. All the stochastic cases with $N>0$ ended the optimization sequence with the same magnetic mirror as the target magnetic field and only the reference case reaches a slightly higher magnetic mirror of $\sim 13 \%$. Unfortunately, the magnetic mirror is not a direct proxy for fast particle confinement which means that a higher magnetic mirror does not necessarily mean better fast particle confinement.

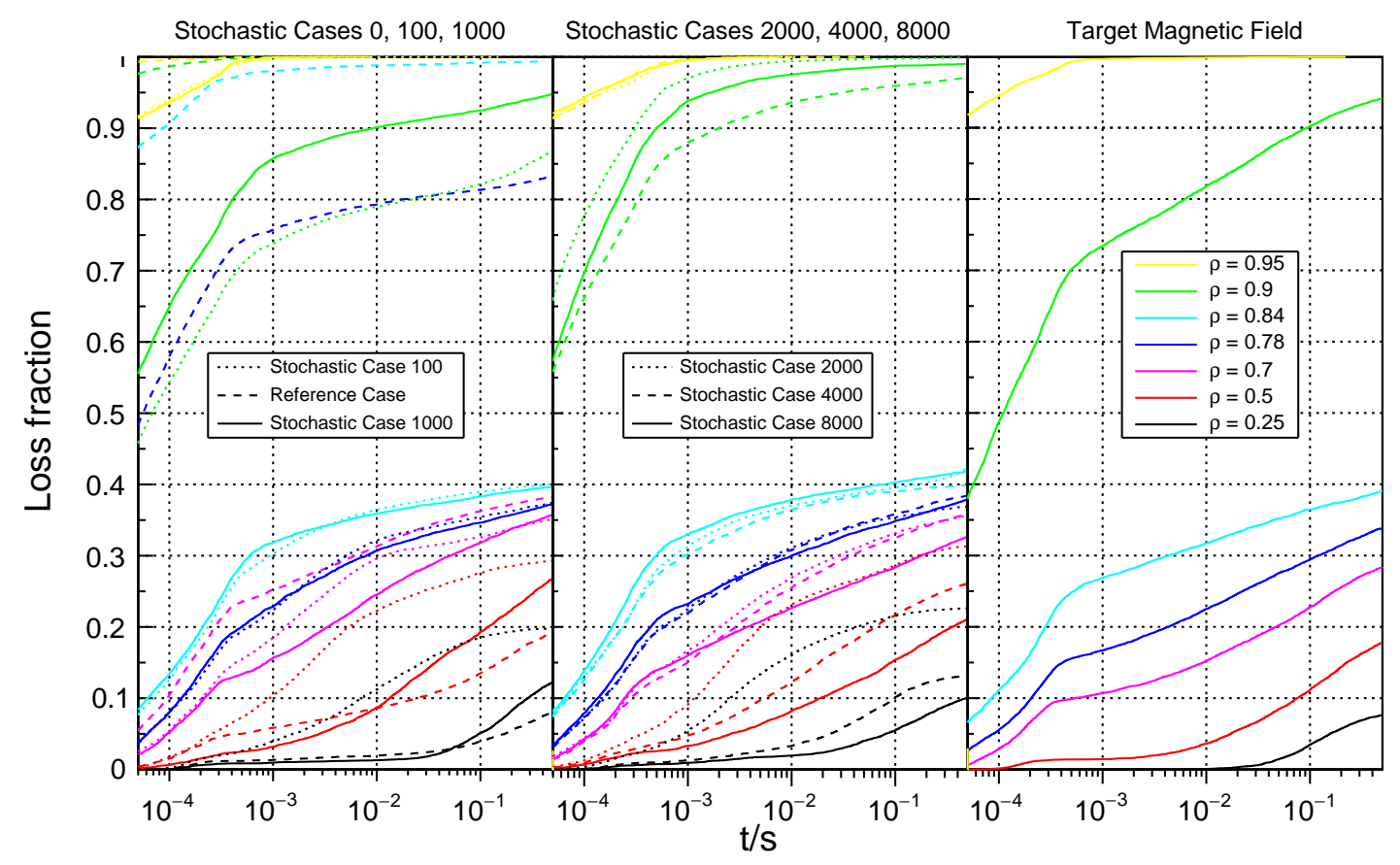

Figure 7: Comparison of the loss fraction of fast particles initiated at different starting positions along the radial direction. The reference case together with the stochastic cases 100 \& 1000 are situated left, the stochastic cases $2000 \& 4000 \& 8000$ are in the middle and the target magnetic field is placed on the right.

We measure the fast particle confinement of the 7 magnetic fields with ANTS [16]. Along the radial direction, 7 different starting positions are defined at $\rho=$ $0.25,0.5,0.7,0.78,0.84,0.9,0.95$. At each radial position an ion population of 10000 particles was initiated and the loss fraction was measured as a function of time. The deuterium ions are initiated with a kinetic energy of $60 \mathrm{keV}$, which yields about the same gyroradius-to-system-size ratio as for fusion alpha particles at $3.5 \mathrm{MeV}$ in a HELIAS reactor [34]. We do not consider collisions while tracing the particles along the field lines in the magnetic field of a $\langle\beta\rangle=5 \%$ equilibrium.

In figure 7 we compare the development of the loss fraction as a function of time starting with the reference case and the stochastic case $100 \& 1000$ on the left, the 


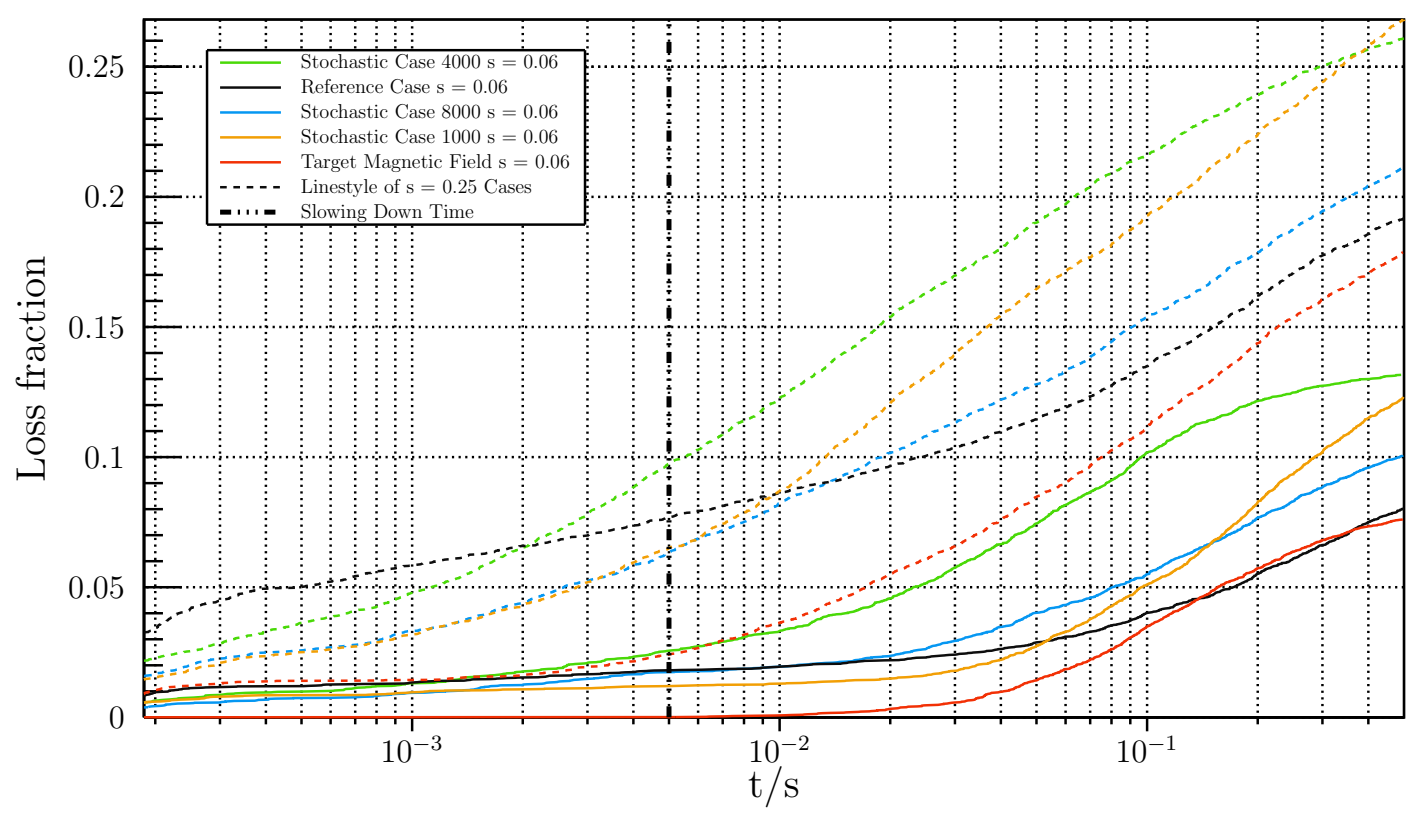

Figure 8: Zoom into the comparison of the loss fraction of the ion populations starting at $\rho=0.25$ and $\rho=0.5$. Additionally, the slowing down time in a similar W7-X magnetic field is indicated.

stochastic cases $2000 \& 4000 \& 8000$ in the middle and the target magnetic field on the right. The latter exhibits excellent fast particle confinement near the axis, as is evident by the small loss fraction for $t<2 \times 10^{-2} \mathrm{~s}$. Details about its optimization, which focused on the confinement close to the axis only, can be found in [11]. During the optimization of the magnetic fields of the stochastic and the reference cases no proxy for the confinement of fast particles was used due to their time-intensive computation. Therefore, the fast particle confinement was only indirectly optimized through the field error.

As expected, the target magnetic field has the best confinement of fast particles at each starting position. If we compare the rest of the cases at $\rho \geq 0.7$, we see that the reference case has noticeably the worst performance while the stochastic cases with $N>0$ have almost the same performance. At $\rho=0.7$ we find, surprisingly, the performance ordered by the number of sample used during the optimization $\left(f_{8000}<f_{4000}<f_{2000}<f_{1000}<f_{100}<f_{1}\right)$. Looking at the two starting positions close to the axis $(\rho=0.25$ and $\rho=0.5)$, we see the worst performance from the stochastic case $100 \& 2000$. A comparison between the remaining 5 cases is shown in figure 8. Additionally, we added the slowing-down time of $5 \times 10^{-3} \mathrm{~s}$ computed for a similar W7-X magnetic field stated in [35]. Its magnetic configuration only reaches a pressure of $\langle\beta\rangle=4 \%$, but the density of $1.6 \times 10^{20} \mathrm{~m}^{-3}$ is comparable to the one shown in section 4.3. A comparison at this particular time step shows that at $\rho=0.25$ the 
fast particle confinement is second best for the stochastic case 1000, closely followed by the stochastic case 8000 and the reference case which have the same performance. At $\rho=0.5$ the stochastic case $1000 \& 8000$ have the second best performance after the target magnetic field with a slightly better performance then the reference case. At both starting positions, the stochastic case 4000 performs worst among the cases considered in figure 8 .

In summary, the fast particle confinement is best in the target magnetic field, which is closely followed by the stochastic cases 1000 \& 8000 which have almost the same performance. Their performance is quite similar to the reference case, but only close to the axis. For $\rho \geq 0.7$ the reference case actually performs worst. Consequently, the performance of the stochastic case 4000 is still better than the reference case which is only better than the stochastic case $2000 \& 1000$ due to their bad performance close to the axis. The final order is:

$$
\text { Target }<f_{8000} \approx f_{1000}<f_{4000}<f_{1}<f_{2000} \approx f_{100}
$$

\subsection{Turbulent Transport}

As neoclassical transport is suppressed, turbulent transport becomes more important in present day stellarators like W7-X [36]. Therefore, attempts have been made to develop models to predict turbulent transport in stellarators in order to optimize future devices [37]. Here, we show simulation results produced by GENE-3D, the radiallyglobal stellarator version of the grid-based gyrokinetic turbulence code GENE [39, 38, 40] which was recently developed at IPP Garching [41]. Previous studies of stellarators with GENE were limited to the flux-tube and flux-surface global version of GENE $[42,43]$. This is one of the first studies performed with the fully global GENE-3D code. We compare three different equilibria mentioned in section 2 w.r.t. to two different temperature and density profiles. The first profile type was used in the investigation of the neoclassical transport of section 4.3 and the second profile type is a standard used in GENE. Its gradient peaks at $\rho=0.5$ and has a Gaussian like shape (see fig. 9). It allows GENE simulations to run accurately with relatively low resolution requirements and hence lower computational cost. The differences of the profiles in location and height of the peak of the gradients can lead to different mode structures in GENE-3D simulations which is part of the upcoming investigation.

4.5.1. Linear simulations with adiabatic electrons Figure 10 shows the most unstable modes drifting in the ion diamagnetic direction in linear simulations assuming Boltzmann-distributed electrons. Looking at the solid lines which correspond to the profiles used in the neoclassical transport investigation of section 4.3, the reference case and the stochastic case 8000 peak at the same toroidal mode number of $n=110$ and the target magnetic field peaks at a slightly smaller mode number of $n=105$. A higher mode number indicates that the underlying mode structure varies on a smaller scale. Large scale instabilities are however more relevant in nonlinear simulations as 


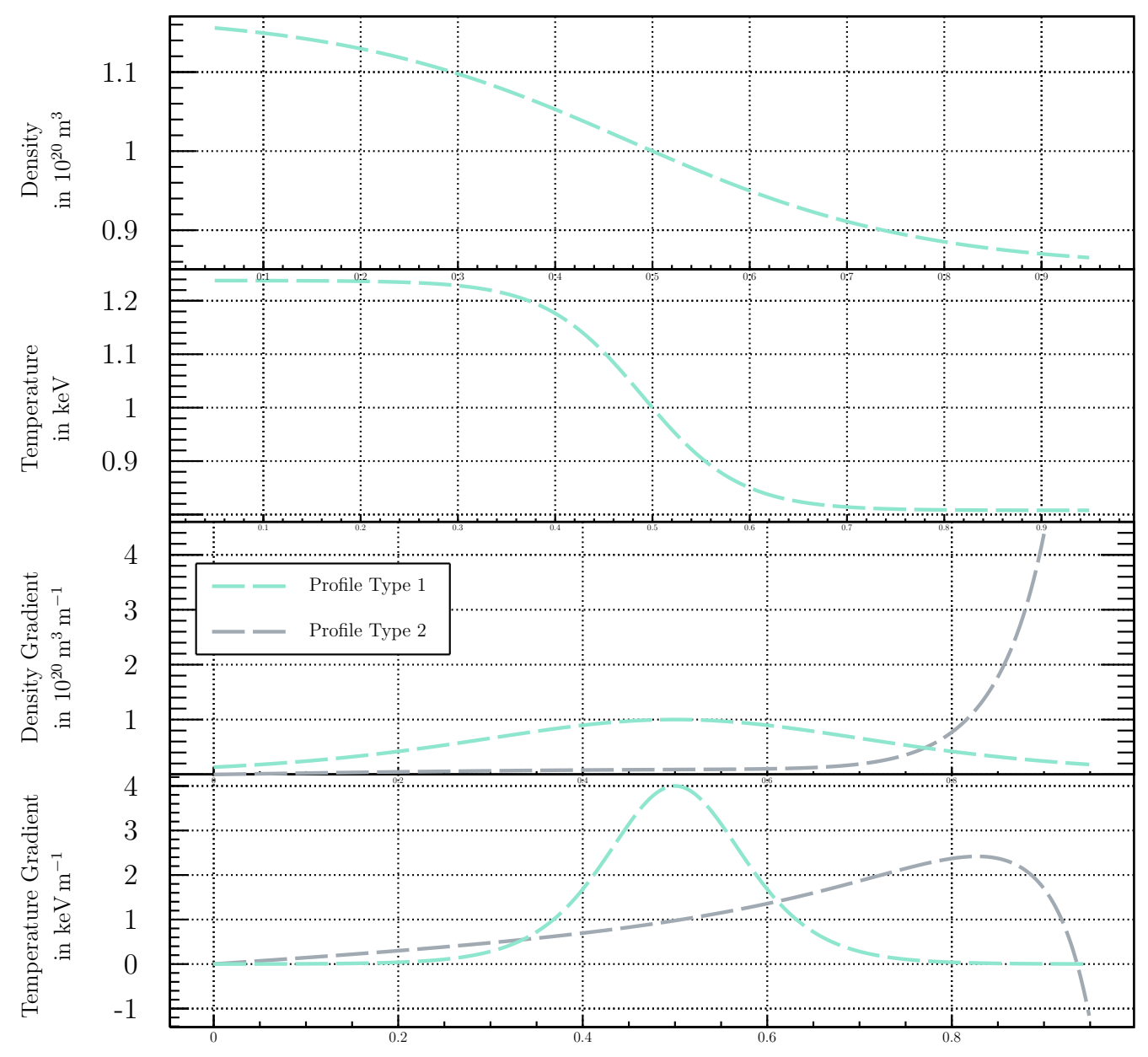

Normalized Minor Radius $\rho$

Figure 9: Density and temperature profiles used in GENE-3D simulations for the mode analysis. The gradient profiles have very different shape and peak at different positions and can therefore lead to different dominant modes in the simulations.

they lead to more particle and heat transport. The picture stays the same when looking at the growth rates, where the target magnetic field has a slightly higher growth rate of $\gamma=0.146 \frac{\mathrm{v}_{\mathrm{i}}}{\mathrm{a}}$ compared to $\gamma=0.137 \frac{\mathrm{v}_{\mathrm{i}}}{\mathrm{a}}$ for the other two cases. Here, $v_{i}$ is the ion thermal velocity defined as $\sqrt{T_{i 0} / m_{i}}$ and $a$ is the minor radius.

Looking at the dashed lines of figure 10 which correspond to the profile type 2 in figure 9 the reference case and the stochastic case 8000 peak again at the same toroidal mode number of $n=175$ but with slightly different growth rates. This time the target magnetic field peaks at a slightly higher toroidal mode number of $n=185$ and has a noticeably higher growth rate of $\gamma=0.202 \frac{\mathrm{v}_{\mathrm{i}}}{\mathrm{a}}$.

As both temperature profiles show similar behavior in respect to differences in the magnetic field geometry we continue the simulations using the profile type 2 which leads to lower computational cost of the GENE-3D simulations. 


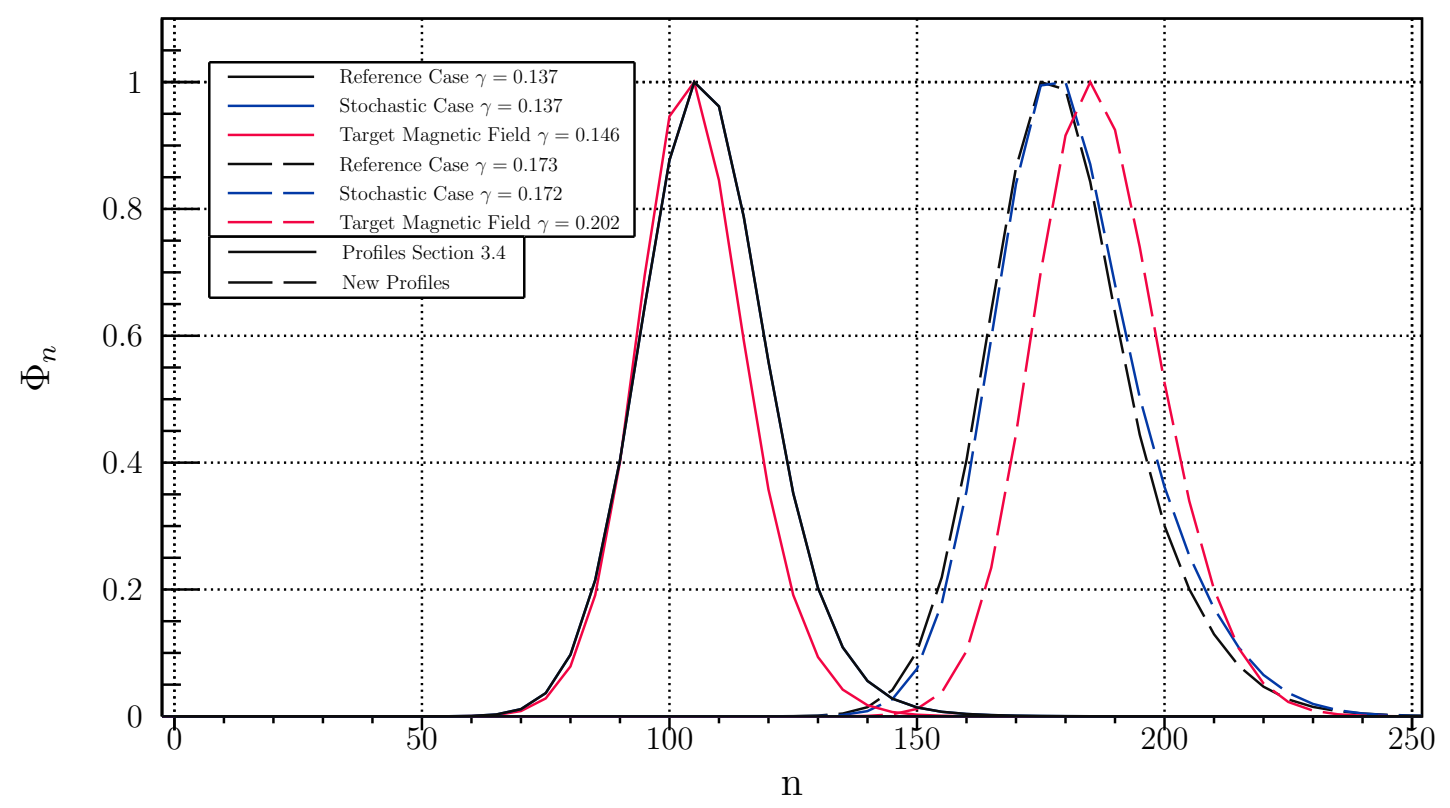

Figure 10: Most unstable modes in linear simulations of adiabatic electrons. Two different temperature and density profiles were used.

4.5.2. Linear simulations with kinetic electrons An explicit electron species can change the outcome of a simulation as the dominant mode can have different characteristics. Theoretically, the mode can change from ion modes such as ion temperature gradient (ITG) modes to electron modes such as electron temperature gradient (ETG) modes or trapped electron modes (TEM). Therefore, we repeated the tests of section 4.5.1 for kinetic electrons to investigate their effect on the mode structure and small changes in the geometry. We assume the same density and temperature gradient for the electrons as for the ions, such that the two linear cases differ only by the additional explicit heavy electron species in the system with $m_{e} / m_{i}=1 / 100\left(m_{e}\right.$ : electron mass, $m_{I}$ : ion mass).

As can be seen from figure 11, adding kinetic electrons to the simulations shifts the toroidal mode number to higher values and increases the growth rate. In the performed simulations with kinetic electrons the dominant mode is still rotating in the ion diamagnetic drift direction like with adiabatic electrons. The up shift in mode number due to kinetic electrons is smallest in the target magnetic field and largest in the reference case. But in general the up shift is smaller than the change of mode number due to the difference in profiles, which indicates that an explicit electron species in the simulations does not have a strong effect on the underlying instability. The reference case again has the lowest growth rate while the target magnetic field has the highest.

4.5.3. Nonlinear simulations with adiabatic electrons Adding kinetic electrons to the simulations in the linear scenario did not have a strong effect on the outcome. 


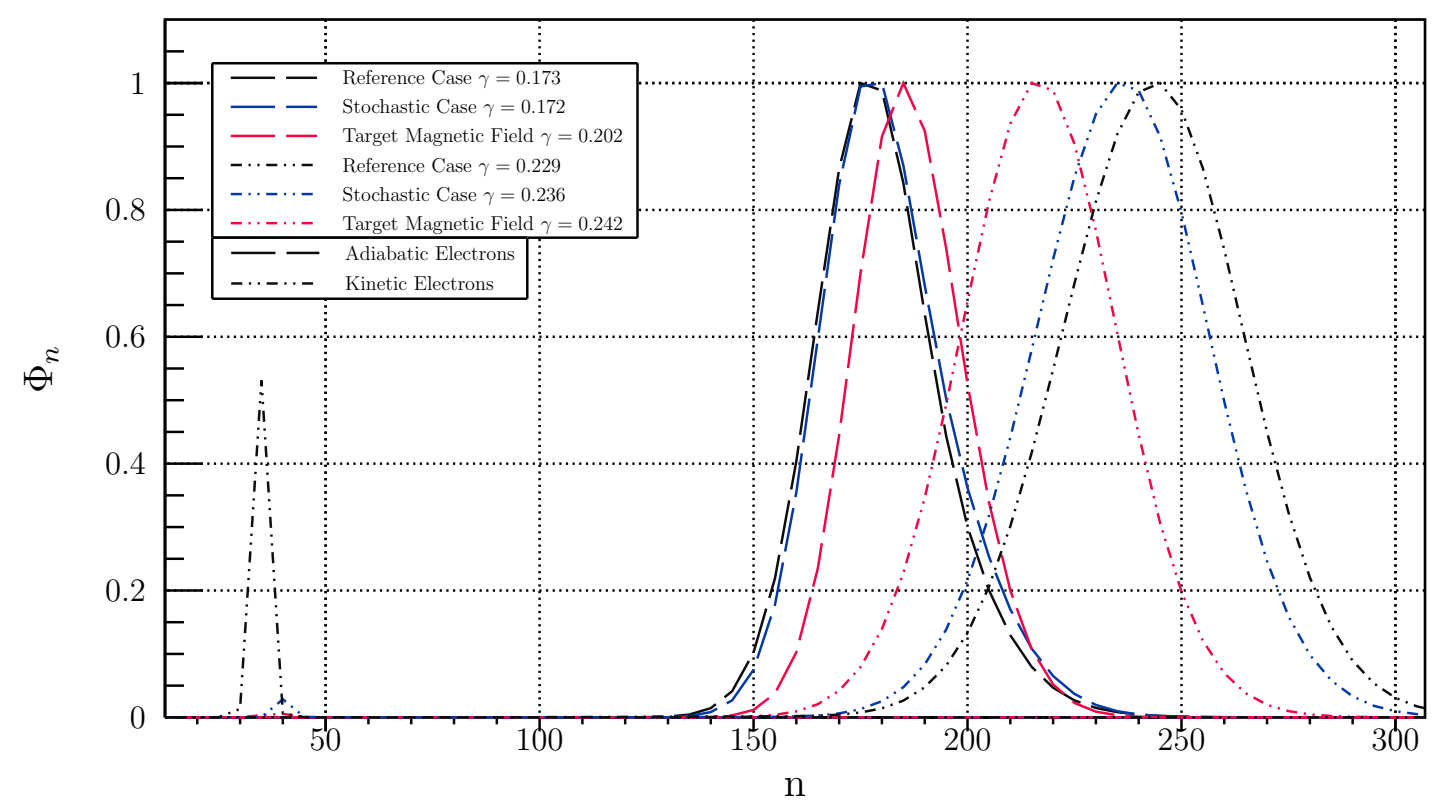

Figure 11: Most unstable modes in linear simulations of kinetic electrons

Therefore, we concentrate on nonlinear simulations of adiabatic electrons to measure the volume-averaged (electrostatic) ion heat flux. As can be seen from figure 12 the three equilibria behave almost identically for the first 250 time units. This confirms the linear results as the first part of virtually uninhibited growth (until $t \approx 100$ ) of a nonlinear simulation is often considered the linear phase until nonlinear effects start to take over. Hereafter, similarity or equality can only be determined statistically because two nonlinear simulations will never be exactly the same. Therefore, the qualitative features of the three equilibria are very similar since they all show the same development. After the first peak follows a dip and then another peak of heat flux. These fluctuations persist even on long time scales. Quantitatively, the heat flux averaged over time for the three equilibria is also very similar as well as their uncertainties. The uncertainty is computed as one standard deviation of the time trace in the interval used for averaging.

4.5.4. Conclusion Comparing three different free-boundary equilibria representing the W7-X high-mirror variant we find that the target magnetic field is actually linearly more unstable than the reference and stochastic case 8000. Between the reference and stochastic case 8000 we could only find minor differences in terms of mode structure or linear growth rate. Nonlinearly, the differences between the three equilibria are negligible. Therefore, considering the performed gyrokinetic simulations small differences in geometry do not affect the heat flux significantly. As the differences between the three different equilibria do not have a large impact on the GENE-3D results such that no additional runs were performed for the rest of the coil cases. 
Total heat transport $Q_{e s}$ over time

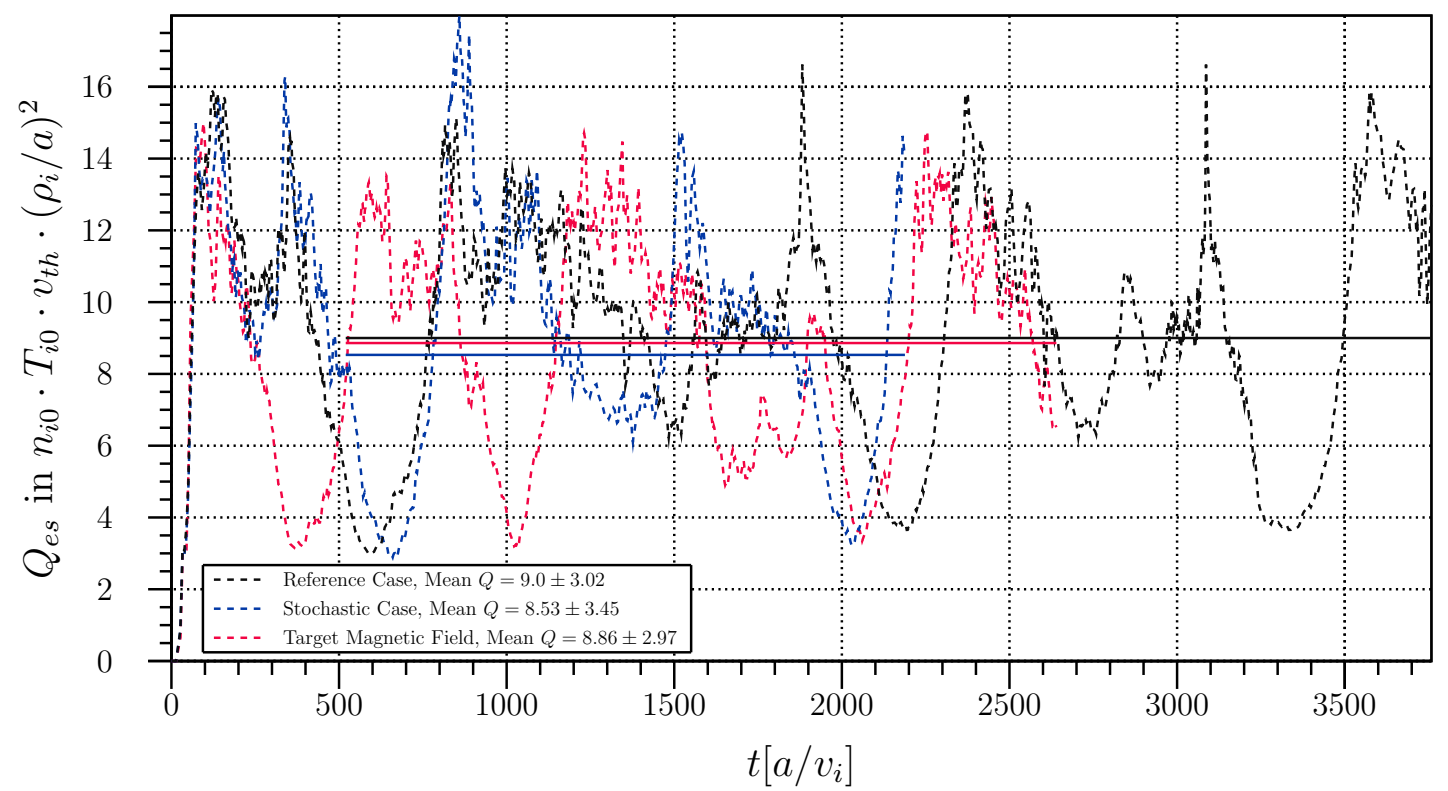

Figure 12: Heat flux development for the three different equilibria. Linear Phase, qualitative features and quantitative averages over time are all virtually the same. Here, $n_{i 0}$ and $T_{i 0}$ are the density/temperature at $\rho=0.5$ and $\rho_{i}$ is the ion Larmor-radius.

\section{Discussion and conclusion}

The magnetic fields of a stochastic stellarator coil optimization (stochastic case $N$ ) are compared with a magnetic field of the traditional stellarator coil optimization (reference case) on the basis of an identical optimization sequence. The results are compared with the target magnetic field used during the optimization. The coil sets are naturally ordered by their fitness w.r.t. the penalty function $f$, which measures the difference between the magnetic field produced by the coil configuration and the target magnetic field by means of several quality criteria. Except for the reference case (sample size 1), the ordering of the penalty values represents the number of samples used during the optimization: The higher the sample size, the lower the penalty value. The reference case lies between the stochastic case 2000 \& 1000 and has a $20 \%$ lower penalty value than the stochastic case 8000 . The stochastic case 100 has by far the highest penalty value, suggesting that it would have the worst performance. Each magnetic field is transformed into a $\langle\beta\rangle=5 \%$ equilibrium with VMEC and compared on the basis of the W7-X objectives [3], namely the Shafranov shift, stability and neoclassical transport properties, and the confinement of fast particles. At last, we investigate the gyrokinetic behavior of the stochastic case 8000, the reference case, and the target magnetic field.

The penalty function $f$ represents the performance of the stochastic cases 8000 \& 4000 \& 2000 together with the reference case quite well when investigating the Shafranov shift, the neoclassical transport and the confinement of fast particles. The 
target magnetic field, which has a penalty value of zero, shows in the latter two criteria the best performance, but only reflects its much better fitness w.r.t. $f$ in the confinement of fast particles. The stability is not very well described by the penalty function and suggests that further improvement should be made. Interestingly, the stochastic cases 1000 \& 100 show good results when investigating the performance criteria which means that the penalty function $f$ did not represent their performance very well. Especially the surprisingly good performance of the stochastic case 100 questions the effectiveness of the penalty function $f$ and shows again that an agreement in the vacuum magnetic field is not necessary for good performance (c.f. [13] and [14]). The investigation of the turbulent transport showed that the differences in the magnetic field geometry are too small to find reasonable differences in the heat flux.

Summarizing the performance investigation, the target plasma shape is optimal followed by the stochastic cases and concludes with the plasma shape of the reference case. This shows that stochastic stellarator coil optimization outperforms classic stellarator coil optimization irrespective of the number of considered samples. The original reason for introducing the stochastic coil optimization was to improve robustness, but it managed to also find coil sets that outperform the previously found coil set even when disregarding tolerances.

\section{Outlook}

The reason why stochastic stellarator coil optimization outperforms classic stellarator coil optimization irrespective of the number of considered samples when analyzing the physics properties of the corresponding magnetic fields is not fully understood. Stochastic optimization smooths out the optimization space which leads to lower field error values when the sample size is high enough. It is left to future work to investigate why stochastically optimized coil configurations with noticeably higher field error values performed better than the reference case.

The fitness ordering obtained from the penalty values after the optimization did not fully reflect the actual expected physics performance of the coil sets. This motivates to invent new proxies especially for the MHD stability. The coil optimization that we have been focusing on here takes as input the target magnetic field and is therefore limited to the performance of the target magnetic field, even if this target itself could be further optimized. Therefore, an obvious next step is to apply the stochastic framework to stellarator optimization, which concentrates on the optimization of the performance criteria directly.

\section{Acknowledgement}

The authors thank V. Perseo, C.D. Beidler, J. Geiger and J. Nührenberg for fruitful discussions and support. This work has been carried out within the framework of the EUROfusion Consortium and has received funding from the Euratom research and 
training program 2014-2018 and 2019-2020 under grant agreement No 633053. The views and options expressed herein do not necessarily reflect those of the European Commission. This work was supported by a grant from the Simons Foundation/SFARI (560651, AB). The simulations presented in this work were performed at the HYDRA and COBRA HPC system at the Max Planck Computing and Data Facility (MPCDF), Germany and at the MARCONI HPC system at CINECA, Italy.

\section{References}

[1] M. Drevlak, 20th Symposium on Fusion Technology, Marseille, France (1998) pp. 883.

[2] J.-F. Lobsien et al., 2018 , Stellarator coil optimization towards higher engineering tolerances, Nuclear Fusion, 58(2018), pp. 106013

[3] Wendelstein VII-X Application for Preferential Support, Wendelstein Project Group, IPPEURATOM Association, AUGUST 1990

[4] P. Merkel, Solution of stellarator boundary value problems with external currents, Nucl. Fusion (1987) 27867

[5] Landreman, 2017, An improved current potential method for fast computation of stellarator coil shapes, Nucl. Fusion, $\mathbf{5 7} 046003$

[6] Paul, E.J., Landreman, M., Bader, A., Dorland, W.: An adjoint method for gradient-based optimization of stellarator coil shapes. Nucl. Fusion 58, 076015

[7] t. Brown et al., 2015, Engineering optimization of stellarator coils lead to improvements in device maintenance, 2015 IEEE 26th Symposium on Fusion Engineering (SOFE), pp 1-6

[8] Zhu et al., 2017, New method to design stellarator coils without the winding surface, Nucl. Fusion, 58016008

[9] H.-S. Bosch et al., Final integration, commissioning and start of the Wendelstein 7-X stellarator operation, Nuclear Fusion, Vol. 57, 116015 (2017)

[10] R.L. Orbach, Statement about the Future of the Princeton Plasma Physics Laboratory, Under Secretary for Science and Director, Office of Science, U.S. Department of Energy, May 22, 2008

[11] Lotz et al., 1990, Optimization, MHD mode and alpha particle confinement behaviour of Helias equilibria, Procs. of 13th Conference IAEA, IAEA-CN-53/C-III-5, p.603

[12] Nührenberg J. and Zille R., 1988 Physics Letters A 129 113-117

[13] Long-Poe Ku, Allen H. Boozer, Stellarator coil design and plasma sensitivity, Physics of Plasmas 17, 122503 (2010)

[14] Zarnstorff M. et al., Physics of the compact advanced stellarator NCSX. Plasma Physics and Controlled Fusion,(2001) 43(12A), A237-A249.

[15] C. Schwab, 1993, Phys. Fluids, B5 3195

[16] Drevlak M., 2009, 36th EPS Conf. on Plasma Physics (Sofia, Bulgaria), P4.211, http://epsppd. epfl.ch/Sofia/pdf/P4_211.pdf

[17] Kisslinger et al., 1990, Magnetic field and coil systems of the modular helias configuration HS 5-10, Fusion Technology, pp. 1520

[18] Hirshman S.P. van Whitson J. C., 1983, Phys. Fluids, 263553

[19] Hirshman S.P. van Rij W.I. and Merkel P., 1986, Comput. Phys. Commun., 43143

[20] C. Nührenberg, Global ideal magnetohydrodynamic stability analysis for the configurational space of Wendelstein 7-X, Phys. Plasmas, 3 (1996) 2401

[21] Strickler, D.J., Berry, L.A., Hirshman, S.P.m Designing Coils for Compact Stellarators. Fusion Sci. Technol. 41, 107-115 (2002).

[22] M. Drevlak, Automated Optimization of Stellarator Coils, Fusion Technology, Vol. 33, No. 2, Page 106-117, 1998

[23] C. Nührenberg, Free-boundary ideal MHD stability of W7-X divertor equilibria, Nuclear Fusion, Vol. 55, 2016, pp. 076010 
[24] Bernstein I.B., Frieman E.A., Kruskal M.D. and Kulsrud R.M., 1958 Proc. R. Soc. A 24417

[25] Beidler C. D. et al., 2011, Nucl. Fusion, 51076001

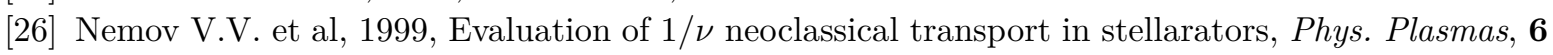
4622

[27] Geiger J., 2015, Physics in the magnetic configuration space of W7-X , Plasma Phys. Control. Fusion, Vol. 57, Nr. 1

[28] Hirshman S.P., Shaing K.C., van Rij W.I., Beasley C.O. and Crume E.C., 1986, Phys. Fluids 29 2951

[29] van Rij W.I. and Hirshman S.P., 1989, Phys. Fluids B 1563

[30] Beidler C. D. et al. "Physics and engineering design for Wendelstein VII-X." Fusion Technology $17.1,(1990), 148-168$.

[31] Dinklage et al., 2007, Assessment of Global Stellarator Confinement: Status of the International Stellarator Confinement Database, Fusion Science and Technology, (51) 1

[32] Turkin Y. et al., 2011, Phys. Plasmas, 18022505

[33] Geiger J., 2007, Stability analysis of Wendelstein 7-X configurations with increased mirror ratio, Proceedings of the 17th International Toki Conference, P. 332-335

[34] T. Sunn Pedersen et al., Key results from the first plasma operation phase and outlook for future performance in Wendelstein 7-X, Phys. Plasmas 24, 2017

[35] S. Äkäslompolo et al., Modelling of NBI ion wall loads in the W7-X stellarator, Nuclear Fusion, 29,2018

[36] Canik, J. et al., Experimental Demonstration of Improved Neoclassical Transport with Quasihelical Symmetry, Phys. Rev. Lett., (98) 8, p.085002, 2007

[37] Hegna, C. et al., Theory of ITG turbulent saturation in stellarators: Identifying mechanisms to reduce turbulent transport, Phys. Plasmas, (25) 2, p.022511, 2018

[38] Görler T. et al., The global version of the gyrokinetic turbulence code GENE, Journal of Computational Physics, 2011, 230

[39] Jenko F. et al., Electron temperature gradient driven turbulence, Physics of Plasmas,(7) 5, p.19041910,2000

[40] Jenko, F. et al., The GENE code, "http://genecode.org", 2019

[41] Maurer M. et al., The 3D global version of the gyrokinetic turbulence code GENE, To be submitted, 2019

[42] Xanthopoulos, P. et al., Controlling Turbulence in Present and Future Stellarators, Phys. Rev. Lett., (113) 15, p.155001, 2014

[43] Xanthopoulos, P. et al., Nonlinear Gyrokinetic Simulations of Ion-Temperature-Gradient Turbulence for the Optimized Wendelstein 7-X Stellarator, Phys. Rev. Lett., (99) 3, p.035002, 2007

[44] Boozer A., What is a Stellarator, Physics of Plasmas, 55

[45] R.C. Grimm, J.M. Greene, J.L. Johnson, Methods of Computational Physics, vol. 9 Academic Press, New York, London (1976), pp. 253-280

\section{Appendices}

\section{Appendix A. Quality Criteria used during the Optimization}

In table A1 we list the achieved values of the quality criteria of the reference \& stochastic cases after the optimization together with the target values. Additionally, we show the corresponding penalty values in brackets (). The quality criteria are grouped in 4 categories and appeared at different stages during the optimization sequence. The 
optimization sequence consisted of 6 optimization runs which differ in the values used for each individual quality criterion. A detailed list of the corresponding weights used in every single optimization run can be found in [2]. The optimization started with the field error together with the geometric properties and introduced the basic properties of the magnetic field in the second stage. The third stage was the optimization of the Fourier coefficients of an inner flux surface. We obtained the Fourier coefficients from the target magnetic field and optimized the corresponding coefficients in the magnetic field produced by the coil set. We use PEST coordinates [45] for this computation which assure identical poloidal angles for the different cases. In the corresponding row in table A1 we are presenting the penalty value instead of the 104 Fourier coefficients. A detailed description of the quality criteria and how they are computed in ONSET [1] can be found in [22]. 
Table A1: Values of the Quality Criteria

\begin{tabular}{|c|c|c|c|c|}
\hline Quality Criteria: & \multicolumn{2}{|r|}{ Stochastic Case 8000: } & arget Values: & Reference Case: \\
\hline \multirow{2}{*}{$\begin{array}{l}\text { Maximum Local Field Error } \\
\text { Average Global Field Error }\end{array}$} & & $6.0 \times 10^{-2}(3.70)$ & (0) & $7.0 \times 10^{-2}(4.87)$ \\
\hline & & $1.6 \times 10^{-2}(1.25)$ & $(0)$ & $1.7 \times 10^{-2}(1.45)$ \\
\hline \multirow{3}{*}{$\begin{array}{l}\text { Curvature } 1\left(\mathrm{~m}^{-1}\right)(\text { Coil } 1 / 2 / 3 / 4 / 5) \\
\text { Curvature } 2\left(\text { in } 10^{-1}\right) \\
\text { Clearance }(\mathrm{cm})\end{array}$} & $3.0 / 2$ & $2.9 / 3.0 / 3.1 / 3.3\left(9.2 \times 10^{-2}\right)$ & $3.0(0)$ & $3.0 / 3.0 / 3.0 / 3.0 / 3.1\left(7.0 \times 10^{-3}\right)$ \\
\hline & $2.7 / 3$ & $3.1 / 5.1 / 6.1 / 6.2\left(1.0 \times 10^{-1}\right)$ & $0.3(0)$ & $3.6 / 4.2 / 5.6 / 5.1 / 5.4\left(6.7 \times 10^{-2}\right)$ \\
\hline & $27 / 27 /$ & $/ 27.7 / 26.8 / 26.8\left(1.4 \times 10^{-3}\right)$ & $27(0)$ & $27 / 26.8 / 26.8 / 26.8 / 31\left(3.1 \times 10^{-3}\right)$ \\
\hline Magnetic Axis (bean-shaped cross.) & & $5.936 \mathrm{~m}\left(5.6 \times 10^{-5}\right)$ & $5.934 \mathrm{~m}(0)$ & $5.928 \mathrm{~m}\left(1.3 \times 10^{-4}\right)$ \\
\hline Magnetic Axis (triangular cross.) & & $5.166 \mathrm{~m}\left(7.2 \times 10^{-3}\right)$ & $5.17 \mathrm{~m}(0)$ & $5.17 \mathrm{~m}\left(1.1 \times 10^{-4}\right)$ \\
\hline Magnetic Mirror on Axis & & $0.10853\left(8.8 \times 10^{-6}\right)$ & $0.107(0)$ & $0.132\left(1.9 \times 10^{-3}\right)$ \\
\hline Iota on Axis & & $0.882\left(9.9 \times 10^{-7}\right)$ & $0.883(0)$ & $0.881\left(3.9 \times 10^{-3}\right)$ \\
\hline Magnetic Shear & & $1.521\left(2.6 \times 10^{-3}\right)$ & $1.56(0)$ & $1.49\left(3.9 \times 10^{-3}\right)$ \\
\hline Magnetic Well & & $8.1 \times 10^{-3}(0)$ & $7.0 \times 10^{-3}(0)$ & $5.4 \times 10^{-3}\left(2.9 \times 10^{-3}\right)$ \\
\hline Fourier Coeff. of inner Flux Surface & & $\left(2.83 \times 10^{-1}\right)$ & (0) & $\left(2.44 \times 10^{-1}\right)$ \\
\hline Total Penalty Value & & $(5.89)$ & (0) & $(6.65)$ \\
\hline \multicolumn{2}{|l|}{ Quality Criteria: } & Stochastic Case 4000: & Stochas & tic Case 2000: \\
\hline \multicolumn{2}{|l|}{ Maximum Local Field Error } & $\begin{array}{c}5.7 \times 10^{-2}(3.29) \\
1.66 \times 10^{-2}(1.39)\end{array}$ & $\begin{array}{c}6.0 \\
195\end{array}$ & $\begin{array}{l}\times 10^{-2}(3.6) \\
\times 10^{-2}(1.9)\end{array}$ \\
\hline \multicolumn{2}{|l|}{ Average Global Field Error } & $\frac{1.00 \times 10-(1.39)}{20(05 / 20 / 201 / 20(40 \times 10-2)}$ & $\begin{array}{r}1.95 \\
-5) \quad 02 / 02 / 01\end{array}$ & $\times 10=(1.9)$ \\
\hline \multicolumn{2}{|c|}{ Curvature $1\left(\mathrm{~m}^{-1}\right)($ Coil $1 / 2 / 3 / 4 / 5)$} & $\begin{array}{l}2.9 / 2.5 / 2.8 / 3.1 / 3.2\left(4.0 \times 10^{-2}\right) \\
2.2 / 2.4 / 4.1 / 5.3 / 5.4\left(5.1 \times 10^{-2}\right)\end{array}$ & $\begin{array}{lr}-2) & 2.2 / 2.3 / 3.1 / \\
-2) & 2 / 3.1 / 4.5 /\end{array}$ & $\begin{array}{l}3.1 / 3.3\left(8.5 \times 10^{-2}\right) \\
5.4 / 5.3\left(5.1 \times 10^{-2}\right)\end{array}$ \\
\hline \multicolumn{2}{|l|}{ Clearance (cm) } & $30.78 / 30.78 / 30.9 / 28 / 28(0)$ & $33.0 / 31.22$ & $29.66 / 27.8 / 27.8(0)$ \\
\hline \multicolumn{2}{|c|}{ Magnetic Axis (bean-shaped cross.) } & $5.922 \mathrm{~m}\left(5.3 \times 10^{-4}\right)$ & 5.923 & $\mathrm{~m}\left(5.0 \times 10^{-4}\right)$ \\
\hline \multicolumn{2}{|c|}{ Magnetic Axis (triangular cross.) } & $5.158 \mathrm{~m}\left(6.4 \times 10^{-2}\right)$ & 5.152 & $\mathrm{~m}\left(1.4 \times 10^{-1}\right)$ \\
\hline \multicolumn{2}{|c|}{ Magnetic Mirror on Axis } & $0.0994\left(1.1 \times 10^{-3}\right)$ & 0.095 & $3\left(3.8 \times 10^{-4}\right)$ \\
\hline \multicolumn{2}{|l|}{ Iota on Axis } & $0.8815\left(6.2 \times 10^{-8}\right)$ & 0.884 & $9\left(5.5 \times 10^{-3}\right)$ \\
\hline \multicolumn{2}{|l|}{ Magnetic Shear } & $1.56\left(9.2 \times 10^{-2}\right)$ & 1.63 & $\left(4.2 \times 10^{-3}\right)$ \\
\hline \multicolumn{2}{|l|}{ Magnetic Well } & $7.9 \times 10^{-3}(0)$ & & $\times 10^{-2}(0)$ \\
\hline \multicolumn{2}{|c|}{ Fourier Coeff. of inner Flux Surface } & $0.646\left(6.46 \times 10^{-1}\right)$ & & $\left..07 \times 10^{-1}\right)$ \\
\hline \multicolumn{2}{|l|}{ Total Penalty Value } & $(5.48)$ & & (6.3) \\
\hline Quality Criteria: & & Stochastic Case 1000: & Stocha & istic Case 100: \\
\hline Maximum Local Field Error & & $7.0 \times 10^{-2}(4.81)$ & $8.0>$ & $\times 10^{-2}(6.38)$ \\
\hline Average Global Field Error & & $1.92 \times 10^{-2}(1.85)$ & $2.27 \times 11$ & $0^{-2}\left(2.58 \times 10^{-2}\right)$ \\
\hline Curvature $1\left(\mathrm{~m}^{-1}\right)($ Coil $1 / 2 / 3$ & $3 / 4 / 5)$ & $2.2 / 2.5 / 3.0 / 3.4 / 3.3\left(1.7 \times 10^{-1}\right)$ & $\begin{array}{l}-1) \\
-1 / 2.4 / 2.6 /\end{array}$ & $/ 3.2 / 3.2\left(3.2 \times 10^{-2}\right)$ \\
\hline Curvature 2 (in $10^{-1}$ ) & & $1.9 / 2.2 / 3.2 / 6.1 / 4.8\left(5.1 \times 10^{-2}\right)$ & 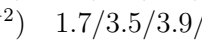 & $/ 4.8 / 5.0\left(4.5 \times 10^{-2}\right)$ \\
\hline Clearance $(\mathrm{cm})$ & & $30.78 / 30.78 / 30.9 / 28 / 28(0)$ & $26.7 / 26 /$ & /26/30.8/34.8 (0) \\
\hline Magnetic Axis (bean-shaped c & cross.) & $5.923 \mathrm{~m}\left(4.8 \times 10^{-4}\right)$ & $5.94 n$ & $n\left(1.6 \times 10^{-4}\right)$ \\
\hline Magnetic Axis (triangular cros & oss.) & $5.174 \mathrm{~m}\left(1.1 \times 10^{-2}\right)$ & 5.191 & $\mathrm{~m}\left(2.2 \times 10^{-1}\right)$ \\
\hline Magnetic Mirror on Axis & & $0.1011\left(8.8 \times 10^{-5}\right)$ & 0.097 & $5\left(2.5 \times 10^{-4}\right)$ \\
\hline Iota on Axis & & $0.879\left(1.2 \times 10^{-2}\right)$ & 0.876 & $8\left(6.6 \times 10^{-2}\right)$ \\
\hline Magnetic Shear & & $1.43\left(1.3 \times 10^{-2}\right)$ & 1.52 & $\left(1.4 \times 10^{-2}\right)$ \\
\hline Magnetic Well & & $1.8 \times 10^{-2}(0)$ & $1.6 \times 1$ & $0^{-2}\left(9.7 \times 10^{-5}\right)$ \\
\hline Fourier Coeff. of inner Flux S & Surface & $\left(7.34 \times 10^{-1}\right)$ & & $(9.62)$ \\
\hline Total Penalty Value & & $(7.65)$ & & (11.03) \\
\hline
\end{tabular}

\section{Description:}


Penalty Value Number in brackets ().

Field Error Normal magnetic field on the plasma boundary.

Clearance Minimum distance between adjacent coils.

Curvature 1 Maximum coil curvature.

Curvature 2 Weighted curvature defined in [22].

Magnetic Axis Central closed field line about which the other field lines wind [44].

Magnetic Shear Difference of iota on axis and an iota value off axis divided by the squared length. 